\title{
Evaluation of strength, agility and aerobic capacity in Brazilian football players
}

\author{
Ana Carolina Gago Raymundo ${ }^{1}$, Carlos Soares Pernambuco ${ }^{1}$, Rosana Dias de Oliveira Brum ${ }^{1}$, \\ Juliana Brandão Pinto de Castro², Flávio Boechat de Oliveira ${ }^{1,2}$, Dirceu Ribeiro Nogueira da Gama², \\ Rodolfo de Alkmim Moreira Nunes ${ }^{2}$, Rodrigo Gomes de Souza Vale ${ }^{1,2}$
}

${ }^{1}$ Laboratory of Exercise Physiology, Estácio de Sá University, Cabo Frio, RJ, Brazil; ${ }^{2}$ Postgraduate Program in Exercise and Sport Sciences, Institute of Physical Education and Sports, Rio de Janeiro State University, Rio de Janeiro, Brazil

\section{Summary}

Study aim: To evaluate the levels of strength, agility and the maximal oxygen uptake $\left(\mathrm{VO}_{2 \max }\right)$ between the offensive and defensive teams of football players.

Material and methods: In the present cross-sectional study, 20 male Brazilian football players were divided into an offensive group ( $\mathrm{OG}, \mathrm{n}=10$, age: $25.50 \pm 6.15$ years) and a defensive group ( $\mathrm{DG}, \mathrm{n}=10$, age: $22.50 \pm 5.48$ years). We used the dynamometer for back and legs, the shuttle run test, and the Cooper test to evaluate strength, agility and $\mathrm{VO}_{2 \max }$, respectively. Results: The independent Student t-test showed that the OG was significantly more agile than the DG (p $<0.05)$. The other variables did not show any statistically significant differences. In the OG there was a strong correlation between agility and $\mathrm{VO}_{2 \max }\left(\mathrm{r}=-0.834, \mathrm{p}=0.003, \mathrm{r}^{2}=0.70\right)$. However, in the DG there was a moderate correlation between the same variables $\left(\mathrm{r}=-0.677, \mathrm{p}=0.031, \mathrm{r}^{2}=0.46\right)$. This shows that the greater the agility is, the higher is the $\mathrm{VO}_{2 \max }$. There was no correlation between the variables muscle strength and body fat percentage.

Conclusions: The study showed that the OG has a better physical condition than the DG.

Keywords: Agility - Muscle Strength - Oxygen Consumption - Maximal oxygen uptake - Athletes

\section{Introduction}

American football is a collective sport practiced in different countries, including Brazil. The game is characterized by the conquest of territories and is composed of two teams, each one with 11 players. Each team is divided into offense and defense, aiming to progress with the ball through the opponent's field (offensive team) and block the opponent's attacks (defensive team). The players of the offense team are: quarterback, center, guards, tackles, wide receivers, running backs and tight ends. The players of the defensive team are: linebackers, ends, defensive tackles, cornerbacks and safeties [25]. American football requires exceptional abilities and the development of physical qualities [26, 40].

Among the physical qualities important for human performance in the practice of football, those that stand out are muscular strength [37] and the agility to move around the field [1]. The oxygen consumption increases during the game, which means that it is also important to develop the aerobic capacity [17], which can improve the ability to tolerate the intensity and duration of the match, and the recovery rate during it [39].

Strength values, in addition to having an individual and genetic component, are also affected by the athlete's sex, age, training level, and sport practiced [12, 38]. The muscle strength measure has been used to improve the results of athletes in competitions and to evaluate the muscular balance in both athletes and non-athletes. Measurement of muscle strength can be performed using methods that use different contraction types: isometric, isokinetic and isotonic [8].

Another important physical quality for football is agility. This is a neuromotor variable characterized by the ability to make rapid changes of direction and displacement of the center of gravity of the whole body or part of it [34], making quick decisions and executing actions efficiently [19]. Several tests and protocols can measure agility. The most common test is the shuttle run [15]. 
The aerobic capacity is usually measured through the maximal oxygen uptake $\left(\mathrm{VO}_{2 \max }\right)[16,33]$. The $\mathrm{VO}_{2 \max }$ is an indicator of the efficiency of the pulmonary and cardiovascular systems, and can be estimated by a conventional exercise test by ergometry and by a field test $[9,27]$. The existing standardizations for the conventional exercise test allow comparisons between individuals. Cardiorespiratory analysis can be classified through different tables, according to age, sex and $\mathrm{VO}_{2 \max }[2]$. The most cited table for the classification of cardiorespiratory fitness is that of Cooper [10].

Accordingly, the offensive and defensive football players can present differences in the levels of strength, agility and $\mathrm{VO}_{2 \max }$. However, few studies have investigated these variables in football athletes and their relations with the positions of these players in a game. Hence, it is important to increase the knowledge about this sport. Additionally, it is important to obtain information on the profile of the physical qualities of these players to help coaches and fitness trainers to prescribe the training with more efficiency [28].

Therefore, the aim of the present study was to evaluate the levels of strength, agility and $\mathrm{VO}_{2 \max }$ among offensive and defensive teams of football players.

\section{Material and methods}

A comparative and cross-sectional study was performed. The study group was composed of 28 football players from the city of Cabo Frio - RJ, Brazil. The inclusion criteria to participate in the present study were to be a practitioner of this sport for at least one year and participate in the State Championship of Rio de Janeiro (Carioca Bowl). Athletes who were, on the day of the data collection, sick, with some injury or muscle discomfort, or who were recovering from an injury, were excluded from the study.

Therefore, three athletes who were recovering from an injury, two who were sick, and three athletes who had other personal problems were excluded from the study. Thus, 20 male subjects were selected and divided into two groups: the offensive group (OG, $\mathrm{n}=10)$ and the defensive group ( $\mathrm{DG}, \mathrm{n}=10$ ).

The players from both groups regularly train three times per week. Each training session lasts 90 minutes. The training is characterized by developing physical, technical and tactical skills. Both groups underwent a diagnostic evaluation, with body mass, height and skinfolds. The subjects could not practice any physical activities on the day of the evaluation.

Each participant signed an informed consent form, outlining the research protocol and possible risks for participating in the study, which is in accordance with the
Declaration of Helsinki and the National Health Council of Brazil.

Throughout the testing period, the recommendations of the International Standards for Anthropometric Assessment from the International Society for the Advancement of Kinanthropometry (ISAK) were used [20]. Height [m] was measured with a stadiometer (Cardiomed, Brazil), with an accuracy of $0.1 \mathrm{~cm}$, and body mass [kg] was measured with a scale (Filizola, Brazil) with a precision of $0.1 \mathrm{~kg}$. To evaluate the skinfolds, the protocol of Jackson and Pollock was used [14]. This protocol has 3 folds: pectoral, abdominal and thigh, measured using an adipometer (Cescorf, Brazil).

In the second stage of the evaluation, the $\mathrm{VO}_{2 \max }$ of the practitioners was analyzed, using the Cooper test on an athletics track, with markers, record sheets and a chronometer (HS-80, Casio, Japan). The volunteer should run or walk for 12 minutes without interruption, aiming to maintain constant speed during the test. The total distance covered during this time was recorded. Based on the distance covered (in $\mathrm{km}$ ), the $\mathrm{VO}_{2 \max }$ was estimated by the following mathematical equation [4]:

$$
\mathrm{VO}_{2 \max }(\mathrm{kg} \cdot \min )^{-1}=(22.351 \times \text { distance })-11.288 \text {. }
$$

To evaluate the agility, the shuttle run test was used, where the individual runs from one line to another, covering a distance of $9.14 \mathrm{~m}$ between the lines. The individual picks up a wooden block, measuring $5 \mathrm{~cm} \times 5 \mathrm{~cm} \times 10 \mathrm{~cm}$, deposited on the ground, and returns to the starting line, placing the object behind it, going to the other line once more, picking up the second block, and also places it behind the line [15]. The test was timed, from start to finish, with a digital chronometer (HS-80, Casio, Japan), with precision of hundredths of a second. The test was repeated twice, with an interval of two minutes between them, using the best result.

For strength testing, a Smedley dynamometer for back and lower limbs (Takey, Japan) was used, where the evaluated subject stood on the dynamometer platform with knees flexed and trunk fully extended. The assessor positioned the head in line with the trunk with the eyes fixed in front and the arms extended. The dynamometer chain was adjusted according to the size of the evaluated subject so that he could hold the support bar, maintaining the position described. The support bar was positioned near the participant's knees. The pointer was placed at the zero position of the dynamometer scale and the participant applied the highest possible force in the knee extension movement [37]. The test was repeated twice with a 1-minute interval, and the best result was taken for the analysis.

\section{Statistical analysis}

The data were analyzed by the IBM SPSS Statistics 20 program and presented as mean, standard deviation and minimum and maximum values. The normality of the data 
was verified through the Shapiro-Wilk test (SW). Student's t-test was used for independent samples for comparison between groups. The Pearson correlation test was used to analyze the possible associations between the study variables. In addition, the effect size $(d)$ was calculated to analyze the magnitude of results. It was interpreted as follows: $<0.2$ : weak; $0.2-0.79$ : moderate; $>0.8$ : strong. The study adopted $\mathrm{p}<0.05$ for statistical significance.

\section{Results}

Table 1 presents the values of the anthropometric characteristics of the analyzed groups. When analyzing this table, it is possible to verify that both groups show a normal distribution. It means that the groups have a normal distribution.

Table 2 shows the comparative results between the groups. The OG was significantly more agile than the DG $(p<0.05)$. The other study variables did not show any statistically significant differences. The strong effect size $(d)$ found in agility demonstrates the magnitude of this variable in the OG. This enhances the results found in this physical quality in the present study. In the other study variables, $d$ was moderate or weak.
Figure 1 presents the correlation between agility and $\mathrm{VO}_{2 \max }$ in the two groups. It can be observed that in the OG there was a strong correlation (shown by the correlation coefficient $\mathrm{r}$ ) between agility and $\mathrm{VO}_{2 \max }$. On the other hand, in the DG there was a moderate correlation between the same variables. This indicates that the greater the agility is, the higher is the $\mathrm{VO}_{2 \max }$. There was no correlation between the variables muscle strength and body fat percentage.

\section{Discussion}

The results of the present study showed a significant difference between the groups in one of the investigated variables. The agility was greater in the OG. In the correlation analysis, the OG showed a stronger correlation coefficient between agility and distance covered $\left(\mathrm{VO}_{2 \max }\right)$.

The values of body fat percentage of the current study did not show significant differences. Other studies have also analyzed this variable and found better levels of physical fitness in athletes with a lower percentage of body fat $[18,31]$.

The variables evaluated in this study, for instance, strength and agility, are important to prevent neuromuscular

Table 1. Characteristics of the offensive group (OG) and the defensive group (DG)

\begin{tabular}{llcccc}
\hline Groups & Variables & Mean $\pm \mathrm{SD}$ & Minimum & Maximum & p-value (SW) \\
\hline OG & Age [years] & $25.50 \pm 6.15$ & 18.00 & 33.00 & 0.335 \\
& Height [m] & $1.78 \pm 0.06$ & 1.70 & 1.87 & 0.063 \\
& Body mass [kg] & $81.17 \pm 22.51$ & 57.10 & 117.00 & 0.152 \\
& Body fat [\%] & $9.99 \pm 4.27$ & 3.86 & 17.54 & 0.724 \\
\hline DG & Age [years] & $22.50 \pm 5.48$ & 18.00 & 35.00 & 0.051 \\
& Height [m] & $1.83 \pm 0.07$ & 1.74 & 1.98 & 0.144 \\
& Body mass [kg] & $82.34 \pm 16.56$ & 61.30 & 114.40 & 0.341 \\
& Body fat [\%] & $11.82 \pm 4.14$ & 4.68 & 19.78 & 0.839 \\
\hline
\end{tabular}

SD - standard deviation; OG - offensive group; DG - defensive group.

Table 2. Comparative analysis of the study variables among sample groups

\begin{tabular}{lcccc}
\hline Variables & Mean $(\mathrm{OG}) \pm \mathrm{SD}$ & Mean $(\mathrm{DG}) \pm \mathrm{SD}$ & $\mathrm{p}$-value & $d$ \\
\hline Strength $[\mathrm{kg}]$ & $162.50 \pm 53.56$ & $168.50 \pm 50.38$ & 0.799 & 0.12 \\
Agility $[\mathrm{sec}]$ & $9.84 \pm 0.42$ & $10.26 \pm 0.30$ & 0.020 & 1.40 \\
Distance $[\mathrm{km}]$ & $2.47 \pm 0.13$ & $2.46 \pm 0.24$ & 0.925 & 0.04 \\
$\mathrm{VO}_{2 \max }[\mathrm{ml} / \mathrm{kg} / \mathrm{min}]$ & $43.85 \pm 2.95$ & $43.66 \pm 5.26$ & 0.925 & 0.04 \\
\hline
\end{tabular}

$\mathrm{SD}$ - standard deviation; OG - offensive group; DG - defensive group; $d$ - effect size. 


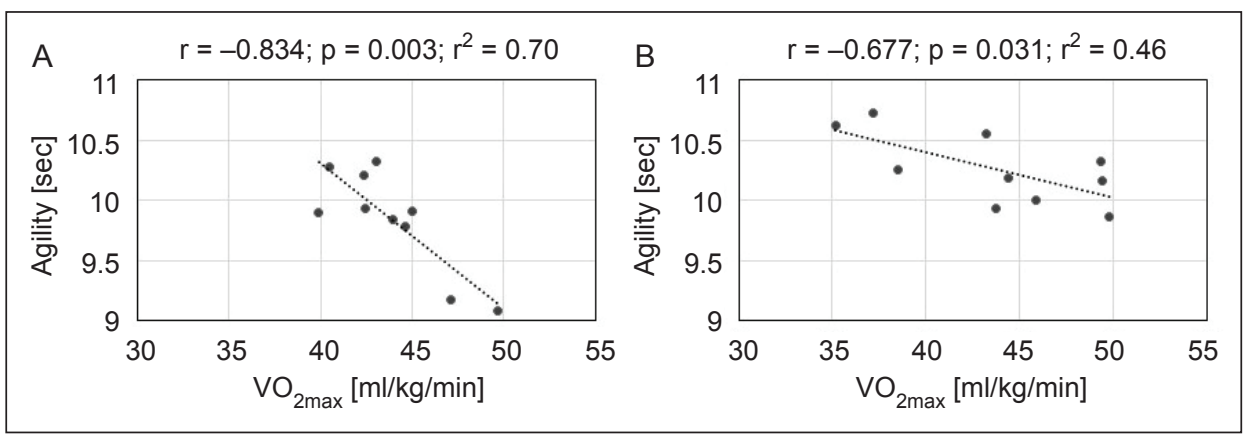

Fig. 1. Correlation analysis between agility and $\mathrm{VO}_{2 \max }$ in the groups. $\mathrm{A}$ - offensive group, $\mathrm{B}$ - defensive group

injuries in team sports [7]. Injuries in sports can cause numerous adverse consequences, such as predisposition to injury in adulthood, technical limitations, and premature end of career [11]. The muscle strength of the football players in the current study showed no statistically significant difference between the OG and the DG. Silveira et al. [36] obtained similar results for this variable, since strength did not show a statistically significant difference between offense (age: $24.5 \pm 4.0$ years) and defense (age: $23.7 \pm 2.8$ years) football athletes.

In the present study, the agility results of the OG were better than those of the DG. This result confirms findings from other studies [1, 21, 35]. This may be associated with the lower body mass of the offensive team, as lighter players tend to be more agile [5]. McGee and Burkett [21] found that agility is an important factor for sport success, and Sierer et al. [35] reported that players who were to be professional had better performance in the agility test compared to players who would not reach that level.

During a football match, over $90 \%$ of the energy expenditure comes from aerobic processes, whose efficiency strongly depends on the aerobic capacity of players, which can be measured through the $\mathrm{VO}_{2 \max }$ [22]. High $\mathrm{VO}_{2 \max }$ levels enhance the post-exercise recovery and utilization of the fat depot as an energy source. It is positive because it allows preservation of muscle glycogen stores [30]. Hence, football players with higher $\mathrm{VO}_{2 \max }$ levels are capable of running longer distances during a match and performing more sprints than those with lower $\mathrm{VO}_{2 \max }$ levels [22].

To calculate the $\mathrm{VO}_{2 \max }$, the present study performed the Cooper test of 12 minutes, resulting in a covered distance in $\mathrm{km}$. There was no difference between OG and DG in this variable. The study of Dinardi et al. [6] compared players of rugby, forwards $(n=9$, age: $23.4 \pm 5.7$ years $)$ and backs ( $\mathrm{n}=9$, age: $30.3 \pm 5.6$ years). The authors also used the Cooper test to measure the $\mathrm{VO}_{2 \max }$. The average distances of forwards and backs were $2.17 \mathrm{~km}$ and 2.16 $\mathrm{km}$, respectively. These values were lower than the $\mathrm{VO}_{2 \max }$ levels found in the groups of the present study. This result may be due to the fact that the rugby players had a shorter training duration than the players of the present study did.

Monteiro et al. [23] used the Yo-Yo test to verify the $\mathrm{VO}_{2 \max }$ in rugby forwards $(\mathrm{n}=13$, age: $24.2 \pm 1.0$ years $)$ and backs ( $\mathrm{n}=12$, age: $23.1 \pm 1.0$ years), with a mean of $42.3 \mathrm{ml} / \mathrm{kg} / \mathrm{min}$ and $47.7 \mathrm{ml} / \mathrm{kg} / \mathrm{min}$ for forwards and backs, respectively. Thus, the rugby backs had superior $\mathrm{VO}_{2 \max }$ levels when compared with the football defense athletes of the present study. This may be due to the nature of rugby, where backs are predominantly required to do constant races with low/medium intensity and cover longer distances.

Sant'Anna and Castro [32] conducted a study with amateur rugby players, comprising 22 forwards and 20 backs. The authors used a laboratory test with a $\mathrm{VO}_{2000}$ gas analyzer, which provides a more accurate result for the calculation of $\mathrm{VO}_{2 \max }$. The mean result of the test in the amateur rugby players was $61.7 \pm 15.0 \mathrm{ml} / \mathrm{kg} / \mathrm{min}$ for the forwards and $51.6 \pm 10.1 \mathrm{ml} / \mathrm{kg} / \mathrm{min}$ for the backs. When compared to the football players of the present study, lower values are observed, which may have been due to the use of different tests. The rugby players performed the test in a laboratory, with control of thermic stress and other external influences, with may also be the cause of the $\mathrm{VO}_{2 \max }$ difference found between the athletes.

It should be noted that, in addition to the anthropometric characteristics and the physical qualities analyzed in the present study, other variables are also adjuvant to athletes' competitive success, such as technical and tactical performance [13], sprint velocity $[22,29]$ and the number of interchanges during a match [3, 24].

This study has a limitation due to the methodological differences that may exist in evaluating the strength, agility and aerobic capacity. Therefore, caution is needed when comparing the results of the present study with other studies. Besides the relevance of the variables evaluated in this study, it is also important to add more variables in future studies based on the specific characteristics needed by football players during a match. 


\section{Conclusions}

The results of the present study demonstrate that the OG of the Brazilian football players of the city of Cabo Frio are significantly more agile than the DG. A strong correlation between agility and the covered distance $\left(\mathrm{VO}_{2 \max }\right)$ was verified in the OG. Therefore, the OG was more physically conditioned than the DG.

It is recommended to carry out specific studies for each position of offense and defense athletes, due to the specific physical predominance for each position. Such knowledge can improve the application of training and, consequently, the performance of football players.

\section{Conflict of interest: Authors state no conflict of interest.}

\section{References}

1. Almas S.P., F.Z. Werneck, E.F. Coelho (2012) Influence of ball carrying on Football players' speed and agility. Motricidade, 8: 682-688.

2. Belli C.K., C. Calegaro, C.M. Richter, J.Z. Klafke, R. Stein, P.R.N. Viecili (2012) Cardiorespiratory fitness of a Brazilian regional sample distributed in different tables. Arq. Bras. Cardiol., 99(3): 811-817.

3. Black G.M., T.J. Gabbett, R.D. Johnston, G. Naughton, M.H. Cole, B. Dawson (2017) The influence of rotations on match running performance in female Australian football midfielders. Int. J. Sports Physiol. Perform. [Epub ahead of print]. DOI: 10.1123/ijspp.2017-0175.

4. Cooper K.H. (1968) A means of assessing maximal oxygen uptake. Correlation between field and treadmill testing. JAMA, 203(3): 201-204. DOI: 10.1001/ jama.1968.03140030033008.

5. Davis D.S., B. Barnette, J. Kiger, J. Mirasola, S. Young (2004) Physical characteristics that predict functional performance in division I college football players. J. Strength Cond. Res., 18(1): 115-120.

6. Dinardi R.R., B.S.P. Aquino, F.C. Ferreira, C.C.F. Bicalho, J.C.B. Marins (2015) Comparative analysis and correlation between different positions of Minas Gerais rugby athletes in relation to physiological measures and physical conditioning. Coleç. Pesqui. Educ. Fís., 14(4): 33-40.

7. Faude O., R. Rössler, E.J. Petushek, R. Roth, L. Zahner, L. Donath (2017) Neuromuscular adaptations to multimodal injury prevention programs in youth sports: a systematic review with meta-analysis of randomized controlled trials. Front. Physiol., 8: 791. DOI: 10.3389/ fphys.2017.00791.
8. Fernandes A.A., J.C.B. Marins (2011) Test of hand grip strength: a methodological analysis and normative data in athletes. Fisioter. Mov., 24(3): 567-578.

9. Hawkins M.N., P.B. Raven, P.G. Snell, J. Stray-Gundersen, B.D. Levine (2007) Maximal oxygen uptake as a parametric measure of cardiorespiratory capacity. Med. Sci. Sports Exerc., 39(1): 103-107. DOI: 10.1249/01. mss.0000241641.75101.64.

10. Herdy A.H., A. Caixeta (2016) Brazilian cardiorespiratory fitness classification based on maximum oxygen consumption. Arq. Bras. Cardiol., 106(5): 389-395. DOI: 10.5935/abc. 20160070.

11. Herdy C., R. Vale, J. Silva, R. Simão, J. Novaes, V. Lima, D. Gonçalves, E. Godoy, J. Selfe, R. Alkmim (2017) Occurrence and type of sports injuries in elite young Brazilian soccer players. Arch. Med. Deporte, 34(3): 140-144.

12. Holm I., N. Vøllestad (2008) Significant effect of gender on hamstring-to-quadriceps strength ratio and static balance in prepubescent children from 7 to 12 years of age. Am. J. Sports Med., 36(10): 2007-2013. DOI: $10.1177 / 0363546508317963$.

13. Hulin B.T., T.J. Gabbett, S. Kearney, A. Corvo (2015) Physical demands of match play in successful and lesssuccessful elite rugby league teams. Int. J. Sports Physiol. Perform., 10(6): 703-710. DOI: 10.1123/IJSPP.2015-0439.

14. Jackson A.S., M.L. Pollock (1978) Generalized equations for predicting body density of men. Br. J. Nutr., 40: 497-504.

15. Johnson B.L., J.K. Nelson (1979) Practical measurements for evaluation in Physical Education. Minnesota: Burgess Publishing.

16. Kaynak K., S.K. Eryılmaz, S. Aydoğan, D. Mihailov (2017) The effects of 20-m repeated sprint training on aerobic capacity in college volleyball players. Biomed. Hum. Kinet., 9(1): 43-50. DOI: 10.1515/bhk-2017-0007.

17. Lambert M., J. Borresen (2010) Measuring training load in sports. Int. J. Sports Physiol. Perform., 5(3): 406-411.

18. Lemos R.S., G.A. Paz, M.F. Maia, J.B. Silva, V.P. Lima, J.B.P. Castro, H. Miranda (2017) Anthropometric and physical fitness parameters versus specific performance tests in Brazilian field hockey athletes: a pilot study. Biomed. Hum. Kinet., 9(1): 57-63. DOI: https://doi. org/10.1515/bhk-2017-0009.

19. Maiewski W., W.C. Souza, T.M. Grelczak, L.P.G. Mascarenhas (2016) Acute effect of neuromuscular proprioceptive and flexing static on agility in American football players. Rev. Fisioter. S. Fun., 5(1): 6-13.

20. Marfell-Jones M., T. Olds, A. Stewart, L. Carter (2006) International standards for anthropometric assessment. Potchefstroom, South Africa: ISAK.

21. McGee K.J., L.N. Burkett (2003) The National Football League combine: a reliable predictor of draft status? J. Strength Cond. Res., 17(1): 6-11. 
22. Michalczyk M., B. Kłapcińska, S. Poprzęcki, S. Jagsz, E. Sadowska-Krępa, E. Kimsa, K. Kempa, J. Chmura (2010) Aerobic capacity and sprint velocity of Leagues I and IV football players. Biomed. Hum. Kinet., 2: 9-14. DOI: $10.2478 / \mathrm{v} 10101-010-0003-0$.

23. Monteiro E.R., A. Mendes, G.A. Paz, H.L. Miranda, V.P. Lima (2016) Aerobic profile between backs and forwards of professional rugby. Rev. Bras. Ciênc. Mov., 24(1): 94-100.

24. Mooney M., S. Cormack, B. O’Brien, A.J. Coutts (2013) Do physical capacity and interchange rest periods influence match exercise-intensity profile in Australian football? Int. J. Sports Physiol. Perform., 8(2): 165-172. DOI: $10.1123 / \mathrm{ijspp} .8 .2 .165$.

25. National Football League. 2017 NFL rule book. United States of America: NFL; 2017. Available from: http:// www.nfl.com/rulebook/beginnersguidetofootball.

26. Nikolaidis P. (2010) Core stability of male and female football players. Biomed. Hum. Kinet., 2: 30-33. DOI: 10.2478/v10101-010-0007-9.

27. Nikolaïdis P.T. (2011) Association between submaximal and maximal measures of aerobic power in female adolescents. Biomed. Hum. Kinet., 3: 106-110. DOI: 10.2478/ v10101-011-0023-4.

28. Nikolaidis P.T. (2014) Evaluation of fatigue in semiprofessional football players: association between overtraining and physical fitness. Biomed. Hum. Kinet., 6(1): 51-55. DOI: 10.2478/bhk-2014-0009.

29. Nikolaidis P.T., B. Knechtle, F. Clemente, G. TorresLuque (2016) Reference values for the sprint performance in male football players aged from 9-35 years. Biomed. Hum. Kinet., 8(1): 103-112. DOI: 10.1515/bhk2016-0015.

30. Ørtenblad N., H. Westerblad, J. Nielsen (2013) Muscle glycogen stores and fatigue. J. Physiol., 591(18): 4405-4413. DOI: 10.1113/jphysiol.2013.251629.

31. Pryor J.L., R.A. Huggins, D.J. Casa, G.A. Palmieri, W.J. Kraemer, C.M. Maresh (2014) A profile of a National Football League team. J. Strength Cond. Res., 28(1): 7-13. DOI: 10.1519/JSC.0000000000000303.

32. Sant'anna R.T., F.A.S. Castro (2017) Aerobic power and field test results of amateur 15-a-side rugby union players. J. Sports Med. Phys. Fitness, 57(12): 1605-1612. DOI: $10.23736 / \mathrm{S} 0022-4707.16 .06574-9$.
33. Sartor F., G. Vernillo, H.M. Morree, A.G. Bonomi, A. La Torre, H.P. Kubis, A. Veicsteinas (2013) Estimation of maximal oxygen uptake via submaximal exercise testing in sports, clinical, and home settings. Sports Med., 43(9): 865-873. DOI: 10.1007/s40279-013-0068-3.

34. Sheppard J.M., W.B. Young (2006) Agility literature review: classifications, training and testing. J. Sports Sci., 24(9): 919-932. DOI: 10.1080/02640410500457109.

35. Sierer S.P., C.L. Battaglini, J.P. Mihalik, E.W. Shields, N.T. Tomasini (2008) The national football league combine: performance differences between drafted and nondrafted players entering the 2004 and 2005 drafts. J. Strength Cond. Res., 22(1): 6-12.

36. Silveira E.M., A. Pincetta, B.F. Marques, L.X.N. Silva, J.L. Teodoro, R.S. Pinto, E.L. Cadore (2017) Avaliação e comparação do perfil físico e das capacidades físicas de jogadores de futebol americano do Rio Grande do Sul. XVII Congresso Brasileiro de Biomecânica.

37. Soares A.V., J.M. Carvalho Júnior, J. Fachini, S.C. Domenech, N.G.B. Borges Júnior (2012) Correlation between handgrip, scapular and lumbar dynamometry tests. Acta Bras. Mov. Hum., 2(1): 65-72.

38. Till K., S. Scantlebury, B. Jones (2017) Anthropometric and physical qualities of elite male youth rugby league players. Sports Med., 47(11): 2171-2186. DOI: 10.1007/ s40279-017-0745-8.

39. Tomlin D.L., H.A. Wenger (2001) The relationship between aerobic fitness and recovery from high intensity intermittent exercise. Sports Med., 31(1): 1-11.

40. Vitale J.A., A. Caumo, E. Roveda, A. Montaruli, A. La Torre, C.L. Battaglini, F. Carandente (2016) Physical attributes and NFL combine performance tests between Italian National League and American football players: a comparative study. J. Strength Cond. Res., 30(10): 2802-2808. DOI: 10.1519/JSC.0000000000001377.

\section{Received 30.10.2017 \\ Accepted 22.01.2018}

(c) University of Physical Education, Warsaw, Poland 